\title{
Diagnostic Efficiency of Adropin As A Preliminary Test To Exclude Acute Pulmonary Embolism
}

\section{Serhat Orun ( $\nabla$ serhatorun@gmail.com )}

Namık Kemal University

Aliye Celikkol

Namık Kemal University

\section{Batuhan Ilbey Basol}

Namık Kemal University

Elif Yeniay

Namık Kemal University

\section{Research Article}

Keywords: Acute pulmonary embolism, adropin, biomarker, emergency medicine.

Posted Date: July 26th, 2021

DOl: https://doi.org/10.21203/rs.3.rs-701497/v1

License: (c) (i) This work is licensed under a Creative Commons Attribution 4.0 International License. Read Full License 


\section{Abstract \\ Background}

This study aimed to investigate the efficiency of adropin as a biomarker to exclude the diagnosis of acute pulmonary embolism (PE).

\section{Methods}

Patients presenting to the emergency department (ED) of a tertiary health center (a university hospital) between August 2019 and August 2020 and diagnosed with PE were included in this prospective cohort study. The amount of serum adropin was determined in patients with PE (who were diagnosed using computerized tomography pulmonary angiography) and compared with healthy volunteers.

\section{Results}

There were 57 participants in the study (28 controls and 29 PE patients). The mean adropin level of the $\mathrm{PE}$ group was $187.33 \pm 62.40 \mathrm{pg} / \mathrm{ml}$ and significantly lower than the control group (524.06 \pm 421.68 $\mathrm{pg} / \mathrm{ml})(\mathrm{p}=0.000)$. When the optimal adropin cut-off value was $213.78 \mathrm{pg} / \mathrm{ml}$, the likelihood ratio of the adropin test was 3.4, and the sensitivity of the adropin test at this value was $82 \%$ with specificity of $75 \%$ (95\% Cl; AUC: 0.821). Further, the highest likelihood ratio obtained was 13.5, where the cut-off value was $304 \mathrm{pg} / \mathrm{ml}$, the sensitivity of the test was $46 \%$, and the specificity of the test was $96 \%$ ( $95 \% \mathrm{Cl}, \mathrm{AUC}$ : $0.821)$.

\section{Conclusion}

The adropin test can be used to exclude the diagnosis of acute pulmonary embolism in the ED. However, more research is required to verify and support the generalizability of our study results.

\section{Introduction}

Acute pulmonary embolism (PE) occurs due to a sudden obliteration of pulmonary arteries, which results from a thrombus ascending up the bloodstream from the deep leg veins [1,2]. The disorder presents with frequent symptoms of sudden dyspnea, stinging chest pain, tachycardia, cyanosis, hemoptysis, and occasional lower extremity swelling with pain [2]. However, the condition is hard to diagnose owing to a lack of specific symptoms and clinical signs. Although PE is an acute life-threatening condition, it is potentially reversible. Therefore, coupled with the development of effective thrombolytic treatments, early and accurate diagnosis of PE is of the utmost importance. 
The gold standard method for the diagnosis of PE is pulmonary angiography, whereas computerized tomography pulmonary angiography (CTPA), which provides similar diagnostic accuracy, is preferred in clinical settings [3]. However, the clinical application of both of the above-mentioned methods has disadvantage due to the associated radiation exposure, the use of radio-opaque materials, and the need for a skilled operator. These disadvantages of radiographic imaging remind the importance of using biomarkers and laboratory facilities for diagnostic purposes. Recent advances in biotechnology have led to the detection of new biomarkers in the laboratory, such as adropin.

Adropin is a polypeptide comprising a total of 76 amino acids, including the first 33 amino acids of the signal peptide, and has a molecular weight of $4999 \mathrm{Da}$ [4, 5]. It is a small polypeptide essentially play an important role in inflammation and some metabolic events [6]. Few studies have suggested the use of adropin as a biomarker in patients suffering from conditions with cellular hypoxia and destruction, such as acute myocardial infarction $[7,8]$.

This study aimed to determine the diagnostic efficiency of adropin to exclude the diagnosis of acute PE, which is characterized by cellular ischemia and necrosis due to obstruction from the embolus

\section{Methods}

Ethical approval for this prospective case-control study was obtained from the Namık Kemal University Ethical Council of Non-Invasive Clinical Researches (registration number - 2019.185.10.06). Convenient patients above the age of 18 years arriving at the emergency department (ED) of a tertiary care center (a university hospital) between August 1, 2019 and August 1, 2020, and diagnosed with pulmonary embolism based on detection of a thrombus in CTPA were included in this study. Patients who were pregnant, had a history of malignancy, were without CTPA imaging, were forwarded to our center with a confirmed diagnosis, and those who required cardiopulmonary resuscitation were excluded from the study. Healthy volunteers of similar age and gender were selected for the comparison group. Written informed consent was obtained from all patients and healthy volunteers who agreed to participate.

Power analysis was performed to determine the sample size. To obtain $80 \%$ power of the analysis at $a=$ 0.05 , a sample size of the PE group and the control group was calculated.

When the diagnosis of PE is confirmed, a blood sample of $5 \mathrm{~mL}$ was drawn from all included patients and put in red capped tubes. The blood tubes were then centrifuged at $3000 \mathrm{rpm}$ for 15 minutes to separate the serum. The serum samples so obtained were kept at $-80^{\circ} \mathrm{C}$. After enough sample size was reached, samples were brought to room temperature on the day of analysis, and serum adropin levels were analyzed using the enzyme-linked immunosorbent assay (ELISA) method. Commercially available kits from Sino Gene Clon Biotech Co., Ltd. (catalog no: SG-11594) were used for the study; the kits have intra-assay and inter-assay CV values of $<8 \%$ and $<10 \%$, respectively.

CTPA imaging was used as the reference standard in this study owing to the similarity of CTPA image results with pulmonary angiography (gold standard for diagnosing PE) and the ease of access to CTPA in 
the ED. The imaging was done using GE Bright Speed model 16 detector, and Mx $0.8 \mathrm{cc} / \mathrm{kg}$ contrast matter was applied under a pressure of 4 psi.

Additionally, age, sex, presenting complaint, the Geneva score and the Wells' score, d-dimer, CRP levels, and arterial blood gas analysis for all patients were obtained and saved in their case report forms.

The laboratory technicians evaluating the adropin test was not informed of the final diagnosis of the patients, clinic findings and the CTPA results. The physician assigned to diagnosing the patients in the ED and evaluating the CTPA images had not have inform by the adropin levels, which were not yet studied.

To evaluate the efficacy of adropin, the blood samples from diagnosed PE patients were compared to healthy volunteers with no active complaints and without any history of chronic disease. The treatment planned for any patient was not changed or retarded during the conduct of the study.

Statistical analyses were performed using Statistical Program for the Social Sciences version 18.0 (IBM Inc.) and Analyse-it (Analyse-it Software Ltd). The Kolmogorov-Smirnov test was used to assess the normality of distribution of the adropin levels and PE parameters, and Student's t-test was used to compare the PE and control groups. Pearson's chi-squared test was used to determine the relationship between gender and emboli variables, and age and emboli variables. Continuous variables were expressed as either mean \pm standard deviation or median (min-max), and the Mann-Whitney $U$ test was used to determine relationships between them. Categorical values were expressed as absolute numbers and percentages. A p-value of $<0.05$ was considered statistically significant. Receiver Operating Characteristic (ROC) analysis was specified with $95 \%$ confidence interval $(95 \% \mathrm{Cl})$ and Area Under Curve (AUC) values. Cut off was determined according to the highest likelihood ratio.

\section{Results}

In this study, $29 \mathrm{PE}$ patients (average age $=64.48 \pm 12.73$ years) and 28 healthy controls (average age $=$ $61.69 \pm 27.22$ years) were included. In the PE group, 19 (65.5\%) were females and 10 (34.5\%) were males, while the control group had 12 females and 16 males. There was no statistically significant difference in age values and sex between the PE and control groups (Table 1). 
Table 1

Comparison of the demographic characteristics of the embolism and control group

\begin{tabular}{|lll|}
\hline & $\mathbf{n}(\%)$ & $\mathbf{p}$ \\
\hline Sex & $19(65.5 \%)$ & 0.07 \\
PE group (Female) & $12(42.8 \%)$ & \\
Control group (Female) & & \\
\hline Age & $64.48 \pm 12.73$ & 0.12 \\
PE group & $61.69 \pm 27.22$ & \\
Control group & & \\
\hline PE; pulmonary embolism & & \\
\hline
\end{tabular}

The presenting complaints on admission for the 29 patients in the PE group were dyspnea $(n=17$, $58.6 \%)$, chest pain $(n=8,27.8 \%)$, weakness $(n=2,6.8 \%)$, tachycardia $(n=1,3.4 \%)$, back pain $(n=1,3.4 \%)$, and cough and hemoptysis $(n=1,3.4 \%$ ) (Table 2). In the PE group, the median value for d-dimer was 5.92 $\mathrm{mg} / \mathrm{L}$ (0.81-35), median CRP levels were $39.5 \mathrm{mg} / \mathrm{L}$ (2.75-239), and median troponin value was $55 \mathrm{ng} / \mathrm{L}$ (4-231). Further, there was no correlation between adropin and d-dimer levels, CRP levels, and troponin levels ( $p=0.2, p=0.2, p=0.2$, respectively). The laboratory results for the PE patients are given in Table 3 .

Table 2

Complaints of PE group admission to the emergency room

\begin{tabular}{|ll|}
\hline Complaints & $\mathrm{n}(\%)$ \\
\hline Dyspnea & $17(\% 58.6)$ \\
\hline Chest pain & $8(\% 27.8)$ \\
\hline Weakness & $2(\% 6.8)$ \\
\hline Tachycardia & $1(\% 3.4)$ \\
\hline Back pain & $2(\% 6.8)$ \\
\hline Syncope & $1(\% 3.4)$ \\
\hline Cough & $1(\% 3.4)$ \\
\hline Hemoptysis & $1(\% 3.4)$ \\
\hline Fever & $1(\% 3.4)$ \\
\hline Epileptic seizure & $1(\% 3.4)$ \\
\hline PE; pulmonary embolism \\
\hline
\end{tabular}


Table 3

Laboratory findings of the PE group

\begin{tabular}{|ll|}
\hline Tests & Mean \pm Std \\
\hline Adropin (PE group) pg/ml & $187.33 \pm 62.40$ \\
\hline Adropin (control group) pg/ml & $524.06 \pm 421.68$ \\
\hline D-dimer $\mathrm{mg} / \mathrm{L}$ & $9.64 \pm 9.95$ \\
\hline CRP $\mathrm{mg} / \mathrm{L}$ & $67.70 \pm 64.38$ \\
\hline Troponin $\mathrm{ng} / \mathrm{L}$ & $54.00 \pm 45.39$ \\
\hline Urea $\mathrm{mg} / \mathrm{dL}$ & $46.04 \pm 29.01$ \\
\hline Creatinine mg/dL & $0.89 \pm 0.25$ \\
\hline WBC $10^{\wedge} 3 / \mathrm{uL}$ & $11.03 \pm 3.78$ \\
\hline Hg g/dL & $12.45 \pm 2.17$ \\
\hline Platelet $10^{\wedge} 3 / \mathrm{uL}$ & $224.75 \pm 87.03$ \\
\hline Ph log[H+]- & $7.45 \pm 0.05$ \\
\hline PO2 mmHg & $69.81 \pm 23.83$ \\
\hline PCO2 mmHg & $35.12 \pm 8.70$ \\
\hline Pt sn & $13.97 \pm 2.68$ \\
\hline Aptt sn & $23.92 \pm 3.50$ \\
\hline INR Inr & $1.21 \pm 0.24$ \\
\hline PE; pulmonary embolism,WBC; whigth blood cell, INR; international normalized ratio \\
\hline
\end{tabular}

Among the 29 PE patients, 3 (10.3\%) were in the low-risk group, 20 (69\%) were in the medium-risk group, and $6(20.7 \%)$ were in the high-risk group according to the Genova risk scoring. The correlation between adropin levels and Genova score was also statistically non-significant $(p=0.5)$. When the same patients were analyzed using the Wells' scoring system, 2 of them (6.9\%) were at low risk, 25 (86.2\%) were at medium risk, and $2(6.9 \%)$ were at high risk of.... The correlation between Wells' score and adropin level was also non-significant $(p=0.3)$ (Table 4). 
Table 4

Risk stratification of PE group according to Wells' and Genova scoring systems

\begin{tabular}{|ll|}
\hline Scoring systems & $\mathrm{n}(\%)$ \\
\hline Genova & \\
\hline Low risk & $3(10.3 \%)$ \\
\hline Medium risk & $20(69 \%)$ \\
\hline High risk & $6(20.7 \%)$ \\
\hline Wells' & \\
\hline Low risk & $2(6.9 \%)$ \\
\hline Medium risk & $25(86.2)$ \\
\hline High risk & $2(6.9 \%)$ \\
\hline PE; pulmonary embolism \\
\hline
\end{tabular}

Both the PE and control groups had normal distribution in terms of adropin levels (Fig. 1). The mean adropin level in the PE group was $187.33 \pm 62.40 \mathrm{pg} / \mathrm{ml}$, compared to $524.06 \pm 421.68 \mathrm{pg} / \mathrm{ml}$ for the control group. The adropin level of the PE group was found to be significantly lower than that of the control group $(p=0.000)$.

When the optimal adropin cut-off value was $213.78 \mathrm{pg} / \mathrm{ml}$, the likelihood ratio of the adropin test was 3.4, and the sensitivity of the adropin test at this value was $82 \%$ with specificity of $75 \%$ (95\% Cl; AUC: 0.821$)$. Further, the highest likelihood ratio obtained was 13.5, where the cut-off value was $304 \mathrm{pg} / \mathrm{ml}$, the sensitivity of the test was $46 \%$, and the specificity of the test was $96 \%(95 \% \mathrm{Cl}$, AUC: 0.821$)$ (Fig. 2).

\section{Discussion}

This study investigates the efficiency of adropin as a biomarker for excluding the diagnosis of acute PE in emergency settings. The results of this study suggest that adropin levels of $\geq 213.78 \mathrm{pg} / \mathrm{ml}$ can be generalized to exclude acute PE with $82 \%$ sensitivity and $75 \%$ specificity. Furthermore, it can be implied that adropin levels of $>304 \mathrm{pg} / \mathrm{ml}$ can exclude a diagnosis of acute PE with $96 \%$ specificity. Serum adropin levels have been indicated to decrease in multiple scenarios and can be evaluated as potential biomarkers in conditions such as diabetes mellitus, arterial hypertension, obesity, sleep apnea syndrome, and even osteoarthritis of the knees [9-12]. Moreover, since adropin levels may be affected by various systemic and non-systemic diseases, we suggest that using higher adropin levels as a preliminary test to exclude acute PE will be beneficial rather than associating lower adropin levels with PE. 
The increased awareness about various venous thromboembolic diseases in recent guidelines, supplemented essentially with an increased availability of non-invasive imaging tests such as CTPA, encouraged clinicians to suspect PE more frequently and start a diagnostic study to confirm the diagnosis at an early stage [3]. Therefore, while evaluating non-invasive diagnostic strategies for PE in recent times, it is essential to exclude PE safely in the present patient population who has a rather low pre-test possibility of having the disease [13]. On the contrary, it is also emphasized that a positive test should have sufficient specificity to determine the indication for treatment, which is anticoagulant treatment in the case of PE [13].

A primary screening or exclusion of patients who are suspected to have developed PE could be conducted in the ED by measuring adropin levels before subjecting the patient to CTPA. This may help decrease the patient's exposure to radiation and the contrast substance. Kaluzna M. et al. reported that adropin levels did not alter significantly during hemodialysis [14], and adropin was described as a potential candidate marker for cardiac dysfunction in patients undergoing hemodialysis. Additionally, Maciorkowska M et al. confirmed a negative correlation of adropin with progression of kidney failure [9]. As seen in our study, there was no acute or chronic kidney failure in the PE group patients, which may have become a contraindication for using contrast substances in these patients. However, in the future with increasing evidence if that suggests that elevated blood adropin is not affected by kidney failure; when patients with limited kidney functions are suspected to develop acute PE, serum adropin may be used.

\section{Limitations}

First, the extent and type of inflammatory involvement in our PE patients, and the influence of inflammation on adropin, was not known. Also, despite excluding patients with a history of malignancy, non-exclusion of patients with other comorbidities and the effect of such comorbidities on adropin levels limits the scope of generalizing our results.

\section{Conclusion}

To conclude, our results suggest that adropin can be used as a preliminary test for excluding the diagnosis of PE. However, since no studies are available that have investigated the efficiency of adropin as a biomarker for PE, we think that the results obtained from our study should not be generalized yet.

\section{Abbreviations}

PE

pulmonary embolism, ED:emergency department, Cl:Confidence interval, AUC:Area under curve, CTPA:computerized tomography pulmonary angiography, ROC:Receiver Operating Characteristic.

\section{Declarations}


Acknowledgements:

The authors are grateful for the help of Namık Kemal University Emergency Medicine workers

\section{Authors' contributions}

SO designed the study, performed the data analysis and drafted the manu-script. AC reviewed and edited the manuscript. BIB performed data acquisi-tion. EY performed data acquisition. All authors read and approved the final manuscript.

\section{Funding:}

The authors declare that they have no funding.

\section{Availability of data and materials}

The datasets used and analyzed for this study are available from the corre-sponding author on reasonable request.

\section{Ethics approval and consent to participate}

This study complied with the Declaration of Helsinki and Ethical approval was obtained from the Namık Kemal University Ethical Council of Non-Invasive Clinical Researches (registration number 2019.185.10.06). Written informed consent was obtained from all recruited patients.

\section{Consent for publication}

Not applicable.

\section{Competing interests}

The authors declare that they have no competing interests

\section{References}

1. Toplis $\mathrm{E}$, Mortimore G. The diagnosis and management of pulmonary embolism. Br J Nurs. 2020; 29(1):22-26. https://doi.org/10.12968/bjon.2020.29.1.22.

2. Righini M, Robert-Ebadi H, Le Gal G. Diagnosis of pulmonary embolism. Presse Med. 2015; 44(12 Pt 2):e385-91. https://doi.org/10.1016/j.lpm.2015.10.007.

3. Konstantinides SV, Meyer G, Becattini C, Bueno H, Geersing GJ, Harjola VP, et al. 2019 ESC Guidelines for the diagnosis and management of acute pulmonary embolism developed in collaboration with the European Respiratory Society (ERS) European Heart Journal. 2020; 41(4):543-603. https://doi.org/10.1093/eurheartj/ehz405 
4. Kumar KG, Trevaskis JL, Lam DD, Sutton GM, Koza RA, Chouljenko VN, et al. Identification of adropin as a secreted factor linking dietary macronutrient intake with energy homeostasis and lipid metabolism. Cell Metab 2008; 8:468-481. https://doi.org/10.1016/j.cmet.2008.10.011

5. Gao S, McMillan RP, Jacas J, Zhu Q, Li X, Kumar GK, et al. Regulation of substrate oxidation preferences in muscle by the peptide hormone adropin. Diabetes 2014; 63:3242-3252. https://doi.org/10.2337/db14-0388

6. Wang B, Xue Y, Shang F, Ni S, Liu X, Fan B, et al. Association of serum adropin with the presence of atrial fibrillation and atrial remodeling. J Clin Lab Anal. 2019; 33(2): e22672. https://doi.org/10.1002/jcla.22672.

7. Aydin S, Eren MN, Yilmaz M, Kalayci M, Yardim M, Alatas OD, et al. Adropin as a potential marker of enzyme-positive acute coronary syndrome. Cardiovasc J Afr. 2017; 28(1):40-47. https://doi.org/10.5830/CVJA-2016-055.

8. Aydin S, Kuloglu T, Aydin S, Kalayci M, Yilmaz M, Çakmak T, et al. Elevated adropin: a candidate diagnostic marker for myocardial infarction in conjunction with troponin-I. Peptides. 2014; 58:91-97. https://doi.org/10.1016/j.peptides.2014.06.001.

9. Maciorkowska M, Musiałowska D, Małyszko J. Adropin and irisin in arterial hypertension, diabetes mellitus, and chronic kidney disease. Adv Clin Exp Med. 2019; 28(11):1571-1575. https://doi.org/10.17219/acem/104551.

10. Gundogdu G, Gundogdu K. A novel biomarker in patients with knee osteoarthritis: adropin. Clin Rheumatol. 2018; 37(8):2179-2186. https://doi.org/10.1007/s10067-018-4052-z. Epub 2018 Mar 16.

11. Choi HN, Yim JE. Plasma Adropin as a Potential Marker Predicting Obesity and Obesity-associated Cancer in Korean Patients with Type 2 Diabetes Mellitus. J Cancer Prev. 2018; 23(4):191-196. https://doi.org/10.15430/JCP.2018.23.4.191. Epub 2018 Dec 30.

12. Bozic J, Borovac JA, Galic T, Ticinovic Kurir T, Supe-Domic D, Dogas Z. Adropin and Inflammation Biomarker Levels in Male Patients With Obstructive Sleep Apnea: A Link With Glucose Metabolism and Sleep Parameters. J Clin Sleep Med. 2018; 14(7):1109-1118. https://doi.org/10.5664/jcsm.7204.

13. Dronkers CEA, Van der Hulle T, Le Gal G, Kyrle PA, Huisman MV, Cannegieter SC, Klok FA. Subcommittee on Predictive and Diagnostic Variables in Thrombotic Disease. Towards a tailored diagnostic standard for future diagnostic studies in pulmonary embolism: Communication from the SSC of the ISTH. J Thromb Haemost 2017; 15:1040-1043. https://doi.org/10.1111/jth.13654

14. Kałużna M, Pawlaczyk K, Schwermer K, Hoppe K, Człapka-Matyasik M, Yusuf Ibrahim A, et al. Adropin and irisin: New biomarkers of cardiac status in patients with end-stage renal disease? A preliminary study. Adv Clin Exp Med. 2019; 28(3):347-353. https://doi.org/10.17219/acem/81538.

\section{Figures}




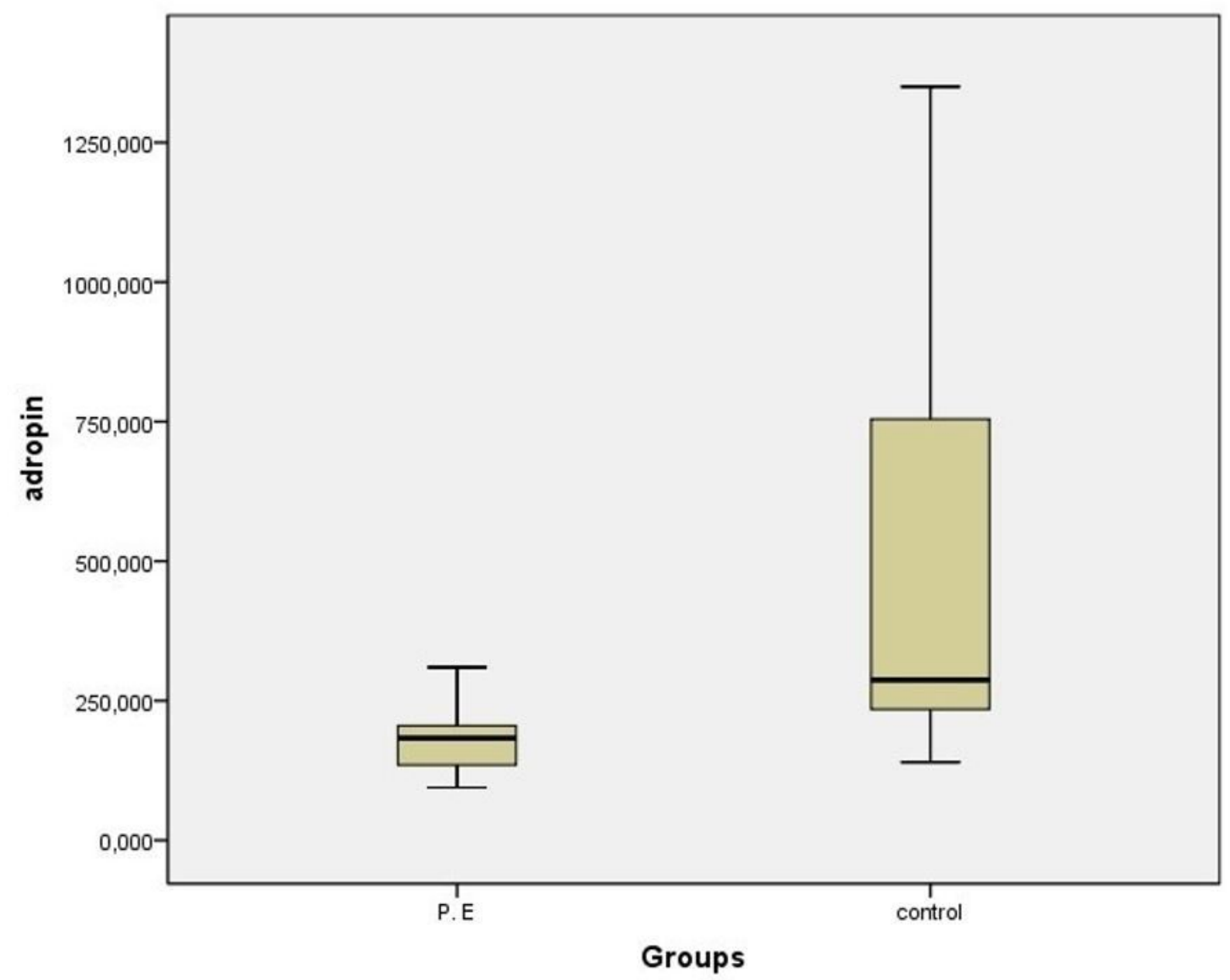

Figure 1

Normal distribution of P.E (pulmonary embolism) and control group adropin values 


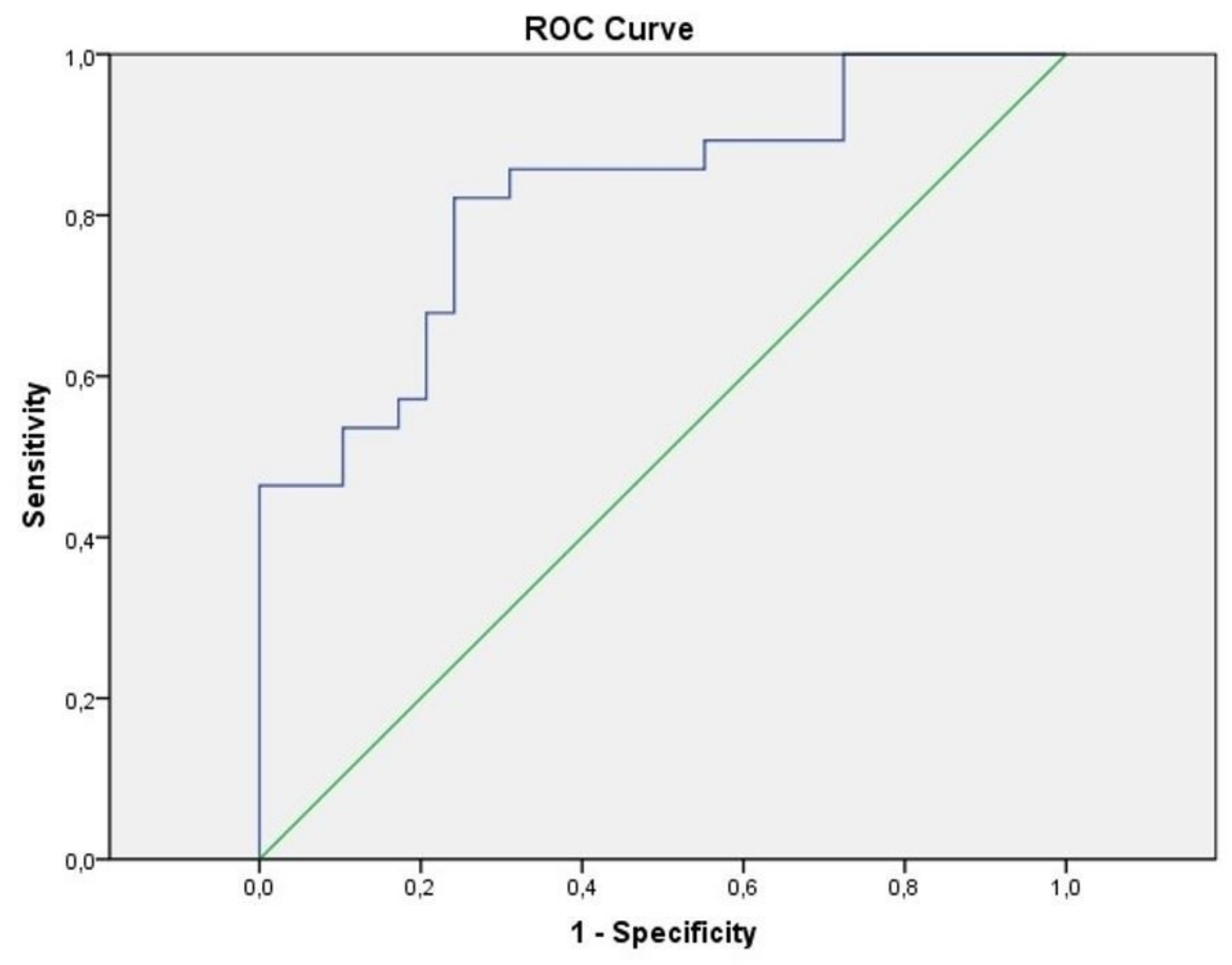

Figure 2

ROC curve of adropine value at excluding pulmonary embolism (blue curve). $95 \% \mathrm{Cl}$, AUC: 0.821 (0,71$0,93)$ 